\title{
Patient-Centered Insights on Treatment Decision Making and Living with Acute Myeloid Leukemia and Other Hematologic Cancers
}

\author{
Rebecca Crawford ${ }^{1} \cdot$ Kate Sully $^{2} \cdot$ Rebecca Conroy $^{1} \cdot$ Chloe Johnson $^{2} \cdot$ Lynda Doward $^{1} \cdot$ Timothy Bell $^{3} \cdot$ Verna Welch $^{3}$. \\ Francois Peloquin ${ }^{4} \cdot$ Adam Gater $^{2}$
}

Published online: 28 August 2019

(c) The Author(s) 2019

\begin{abstract}
Expectations relating to treatment and survival, and factors influencing treatment decisions are not well understood in adult patients with acute myeloid leukemia. This study analyzed combined findings from a targeted literature review with patientreported information shared on YouTube to further understand patient perspectives in hematologic cancers and, in particular, acute myeloid leukemia. The targeted literature review included articles concerning patient (aged $\geq 18$ years) experiences or perspectives in acute myeloid leukemia or other hematologic cancers. YouTube video selection criteria included patients (aged $\geq 60$ years) with self-reported acute myeloid leukemia. In total, 26 articles (13 acute myeloid leukemia-specific and 14 other hematologic cancers, with one relevant to both populations) and 28 videos pertaining to ten unique patients/caregivers were identified. Key concepts reported by patients included the perceived value of survival for achieving personal and/or life milestones, the emotional/psychological distress of their diagnosis, and the uncertainties about life expectancy/prognosis. Effective therapies that could potentially delay progression and extend life were of great importance to patients; however, these were considered in terms of quality-of-life impact and disruption to daily life. Many patients expressed concerns regarding the lack of treatment options, the possibility of side effects, and how their diagnosis and treatment would affect relationships, daily lives, and ability to complete certain tasks. Both data sources yielded valuable and rich information on the patient experience and perceptions of hematologic cancers, in particular for acute myeloid leukemia, and its treatments. Further understanding of these insights could aid discussions between clinicians, patients, and their caregivers regarding treatment decisions, highlight outcomes of importance to patients in clinical studies, and ultimately, inform patient-focused drug development and evaluation.
\end{abstract}

\section{Introduction}

Acute myeloid leukemia (AML) is one of the most prevalent types of blood malignancies, although overall it is a rare orphan malignancy [1-3]. The incidence of AML increases with age, with the mean age at diagnosis between 65 and 70 years [1-3]. Remission rates in older patients (aged $\geq 60$ years) are lower and relapse rates are higher, typically resulting in death within weeks or months of diagnosis [4, 5]. The median survival of patients with AML ranged from 6.2 to 11.0 months, and survival decreased with increased

Electronic supplementary material The online version of this article (https://doi.org/10.1007/s40271-019-00384-9) contains supplementary material, which is available to authorized users.

Timothy Bell

timothy.j.bell@pfizer.com

Extended author information available on the last page of the article

\section{Key Points}

Both the targeted literature review and YouTube video searches provided complementary and valuable information on the patient experience and perceptions of hematologic cancers, in particular for acute myeloid leukemia.

Key concepts reported by patients included the perceived value of survival, the emotional/psychological distress of their diagnosis, the availability and impact of treatment options, and uncertainties about life expectancy/ prognosis.

The patient experience is complex and multifactorial, thus patient management and treatment decisions in clinical practice should be made jointly between patients and clinicians, and need to reflect the expectations, goals, and preferences for a given individual. 
age [6-11]. In a Surveillance, Epidemiology, and End Results (SEER) database analysis from 1997 to 2006, 5-year relative survival rates decreased with increasing age in male and female patients, respectively: $21.1 \%$ and $24.3 \%$ of all ages, $6.8 \%$ and $9.3 \%$ aged $65-74$ years, $1.5 \%$ and $1.1 \%$ aged $75-84$ years, and $1.2 \%$ and $0 \%$ aged $\geq 85$ years [12].

For older patients with AML, there is no standardized treatment pathway; there is a reluctance to treat the older patient population with intensive chemotherapy, with clinicians favoring less intensive chemotherapy or best supportive care [2]. In a population-based study in the USA, only $38.6 \%$ of 5480 patients with AML received chemotherapy within 3 months of diagnosis [13]. Older patients have a higher incidence of comorbidities and adverse molecular and cytogenetic abnormalities, and are at a higher risk for treatment-related morbidity and mortality, all of which contribute to a generally poorer prognosis [2, 3, 14]. As well as impacting treatment decisions, these clinical factors may negatively affect health-related quality of life (HRQoL) [15].

The assessment of HRQoL has become increasingly important in oncology; however, HRQoL in hematology, and specifically AML, has received relatively little attention [16-19]. Furthermore, there is little published information on the perspectives of older patients with AML in terms of their expectations for treatment and survival, and which non-clinical factors influence their treatment decision-making process. More recently, there has been a rise in patient involvement and engagement in clinical research, and patient-centered information is becoming increasingly important in healthcare decision making [20, 21]. Patient-reported information and patient-reported outcomes are forms of patient-centered information. A patientreported outcome measure assesses one or more aspects of a patient's health status (e.g., HRQoL, symptoms, treatment satisfaction) based on information gathered directly from the patient, without interpretation by clinicians or others, and can be utilized in clinical trials and clinical practice [21]. Patient-reported information is experiences reported by patients and caregivers regarding the disease and treatments outside the constraints of formal research, including clinical trials, and in an unsolicited manner [21].

Draft guidance from the US Food and Drug Administration advocates the value of social media as a complementary data-collection method during the preliminary stages of a research study, or as a supplement to traditional research methods such as literature reviews and surveys [22]. In particular, the US Food and Drug Administration guidance highlights social media searches as a method to gain insight on the patient perspective of symptoms and disease impact [22]. Compared with traditional methods, social media data can provide a large-scale, rapid, and cost-effective means to capture patient-reported information in an easily accessible manner [21, 23-25]. Although social media data are generally self-reported, have no interviewer bias, and have fewer resource burdens vs. traditional sources, data can be inconsistent and limited. Furthermore, there remains some uncertainty in the representativeness of the information being reported on social media [21,23-25]. Varying results have been reported in other therapy areas where YouTube was utilized for communicating health and medical treatment information [26-30].

To further understand patient perspectives with regard to treatment decision making and the value of extended life vs. quality of life $(\mathrm{QoL})$, data were obtained from a targeted literature review as well as publicly available patient-reported information shared on YouTube. A particular focus of these analyses was to gain insights from patients with AML on their disease experience and treatment perceptions; however, this was expanded to include other hematologic cancers, owing to the paucity of research in this condition. Additionally, the study aimed to preliminarily assess the feasibility of using YouTube as a supplementary method for collecting patient-reported information in patients with hematologic cancers, in particular, patients with AML.

\section{Methods}

\subsection{Targeted Literature Review}

Searches of computerized bibliographic databases-PubMed (MEDLINE), EMBASE, and PsycINFO-were conducted to identify relevant publications using the OVID platform and a combination of keywords and Medical Subject Headings (MeSH) terms. A focused search strategy applied a combination of population-specific, broad HRQoL/QoL, qualitative research and value of extended life terms (see Electronic Supplementary Material [ESM]). The primary focus of this preliminary search was to explore existing research in patients with AML. Following the initial searches in OVID, an additional search was conducted to include other hematologic cancers, e.g., chronic myeloid leukemia and multiple myeloma (see ESM). Supplementary searches were also performed in Google Scholar. Published guidance and conference proceedings from major hematology and oncology congresses (American Society of Hematology, American Society of Clinical Oncology, Bloodwise, European Hematology Association, and European Society for Medical Oncology) were also reviewed.

The titles and abstracts of the articles generated by searches were reviewed to identify relevant publications (journal articles vs. conference abstracts and excerpts). To be eligible for inclusion, articles were required to have a relevant clinical term included in the title and/or abstract (e.g., 'AML' or 'Acute Myeloid Leukemia'), the main focus 
of the article on patient experience or perspectives, and the study population $\geq 18$ years old. Articles were published within the past 15 years (range 2003-2018). Abstracts not related to the value of extended life in patients with hematologic cancers or their caregivers, or that were unavailable in English, were excluded.

The following information was extracted from each article: author, title, publication type, study objectives, study population, and study design. The value of living longer was explored from the patient and caregiver perspectives, for example, by using descriptions of target or expected life milestones. Qualitative descriptions from the patient, caregiver, and healthcare professional perspective around treatment decisions were also extracted. Finally, and where possible, other relevant factors related to treatment decision making, i.e., evidence of transfusion independence, frequency of clinic or hospital visits, location of treatment center, and incidence of infections, were obtained from each article.

\subsection{YouTube Analysis}

YouTube was selected as it is a global online platform where registered users can easily upload and share videos. Videos that are uploaded with "public" privacy settings can be viewed by any Internet user. According to the YouTube Press statistics, YouTube has over a billion users; nearly a third of all internet users. Furthermore, YouTube has previously been used for conveying health information in a number of therapy areas [26-31]. Finally, YouTube was selected because it is a widely used and well-known platform, familiar to a broad range of age groups. This was in line with the study objectives on investigating the feasibility of a general social media platform for accessing patient-reported information.

Video data were collected via YouTube. Searches focused on identifying videos uploaded by patients with AML or their family members within the past 3 years. For ease of reporting, family members are referred to as 'caregivers' for the YouTube analyses. An initial search (Stage 1) focused on patients aged $\geq 60$ years or older who were ineligible for intensive chemotherapy. This criterion was expanded in the secondary search (Stage 2) to include patients aged $\geq 60$ years who were receiving intensive chemotherapy. This decision was made to increase the number of available videos for review and to further inform on the decision-making process for accepting or rejecting chemotherapy. To identify potentially relevant videos, searches were conducted in an iterative manner using key search terms singularly and in combination, as appropriate (see ESM).

Video screening was conducted in three steps: screening for relevance (Step 1), screening for patient characteristics (Step 2), and video review (Step 3). Step 1 involved manual screening to remove irrelevant videos, e.g., clinician presentations or marketing videos. In Step 2, further manual screening was performed to identify videos that met key patient characteristics. Patients were $\geq 60$ years of age, either self-reported or researcher determined. Step 2 consisted of a staggered approach with an initial search (Stage 1) to identify patients who were ineligible for intensive chemotherapy treatment and a secondary search (Stage 2) to include patients receiving intensive chemotherapy. Stage 1 criteria for patients ineligible for intensive chemotherapy were level of unfitness (either self-reported or patient report of clinical evaluation that would exclude them from intensive chemotherapy) or patient-reported use of any of the following treatments: low-dose cytarabine $\left(\right.$ Cytosar- $\left.\mathrm{U}^{\circledR}\right)$, azacitidine $\left(\operatorname{Vidaza}^{\circledR}\right)$, decitabine $\left(\right.$ Dacogen $\left.{ }^{\circledR}\right)$, palliative care, end-of-life care, allopathic medicine, or non-intensive treatment. The Stage 2 criterion was patient-reported use (current or previous) of intensive chemotherapy treatment. In Step 3, video footage was reviewed to identify the relevance of the content to address the study research questions. Selected videos were retained for in-depth review and analysis.

Because social media patient-reported information exists outside the research context, key patient demographic and diagnostic characteristics were not always available and age was not consistently reported on YouTube videos. If age was not reported, researchers judged approximate age. Content was analyzed thematically to address the key research questions on treatment decisions and preferences.

\subsection{Ethics and Reporting of Results}

Prior to data collection, one of RTI International's institutional review boards reviewed the YouTube study and determined the social media review did not constitute clinical research with human subjects. Ethics approval was deemed unnecessary as the study data were located within the public domain. Ethics approval was not required for a literature review study.

For the purposes of reporting the results, the key themes emerging from both the targeted literature review and YouTube search were combined. Additionally, as the same search criteria were used, the findings from patients with AML and those with other hematologic cancers were combined.

\section{Results}

\subsection{Literature Review}

In the targeted literature review, 1021 abstracts were identified from the search of AML-specific articles (Fig. 1). No 
additional papers were identified through supplementary searches, e.g., Google Scholar and conference proceedings. Fourteen abstracts were selected for full-text review, comprising 11 journal articles and three conference abstracts. All articles were published within the past 15 years (range 2003-2018). One article focused on older patients with AML who were not receiving intensive chemotherapy and the remaining articles focused on patients with AML who were eligible for chemotherapy. One article was excluded because the findings were focused on the presentation of symptoms experienced by patients with AML.

In the expanded supplementary search of other hematologic cancers, 6445 abstracts were identified (Fig. 1). Searches were conducted only within the OVID platform and review articles and those focused on clinical trials were excluded. Sixteen abstracts were selected for full-text review; all were journal articles that had been published between 1986 and 2018. Of these 16, two papers were later excluded as the findings did not meet the objectives of the literature review. The papers covered a range of hematologic cancers, including lymphoma $(n=11)$, myeloma $(n=7)$, and leukemia $(n=6)$. Some papers included patients with nonhematologic cancers $(n=3)$; however, all papers contained at least one participant in the study population who had a hematologic cancer.

A total of 26 articles were selected for inclusion in the targeted literature review synthesis: 13 AML-specific and 14 other hematologic cancers, with one article with findings relevant to both populations [32-57]. Most articles selected for inclusion were qualitative research studies $(n=23)$, providing rich detail regarding the patient perspective.

\subsection{YouTube Search}

In the YouTube search, 49 videos were identified and comprised a total of $3 \mathrm{~h}, 52 \mathrm{~min}$ of video footage (Fig. 2). Twenty-one videos ( $60 \mathrm{~min}$ of video footage) were excluded and the analysis included 28 videos $(2 \mathrm{~h}, 52 \mathrm{~min}$ of video footage) relating to ten unique patients with AML. Of the ten patients or caregivers, eight were patients with AML reporting on their personal AML experience. One was a patient who uploaded a video along with his wife (both of whom provided information on the patient's AML experience) and one was a deceased patient whose wife had uploaded a video reporting on her late husband's experience. Six of the patients were male and ages ranged from 60 to 80 years. The majority of videos $(n=21 ; 75.0 \%)$ had been uploaded to YouTube within the past 3 years. The mean video duration was $6 \mathrm{~min}, 9 \mathrm{~s}$ (range $2 \mathrm{~min}, 28 \mathrm{~s}$ to $14 \mathrm{~min}, 1 \mathrm{~s}$ ). Eighteen (64.3\%) videos had been self-published on YouTube by the patient; the remaining ten videos had been posted by medical centers/organizations ( $n=5 ; 17.9 \%)$, commercial/ pharmaceutical companies $(n=3 ; 10.7 \%)$, and charities/ patient advocacy groups $(n=2 ; 7.1 \%)$. The videos posted by commercial/pharmaceutical companies included a disclaimer stating that the patient testimonial was homemade by the patient and the unedited video had been posted on their behalf.

The key themes emerging from both data sources, and in patients with AML and other hematologic cancers, were combined to inform on the disease experience and associated treatments from the patient perspective.
A

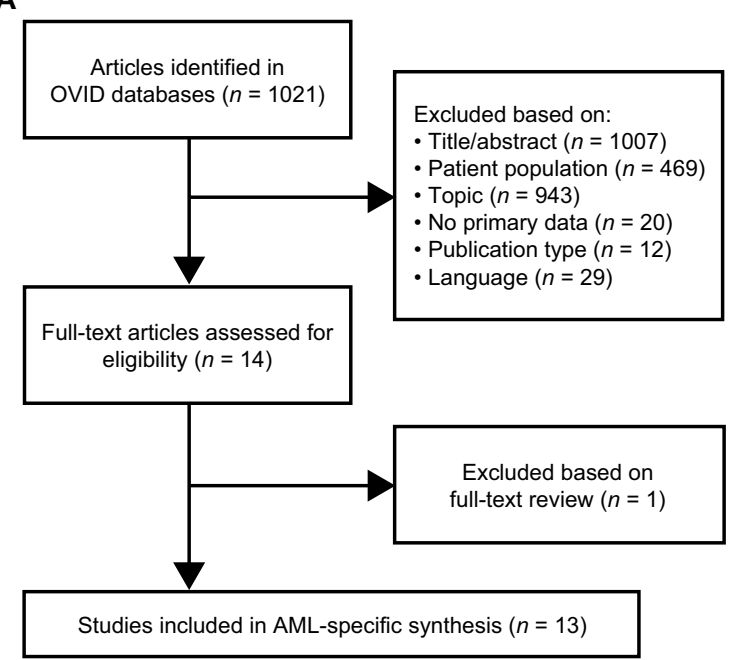

B

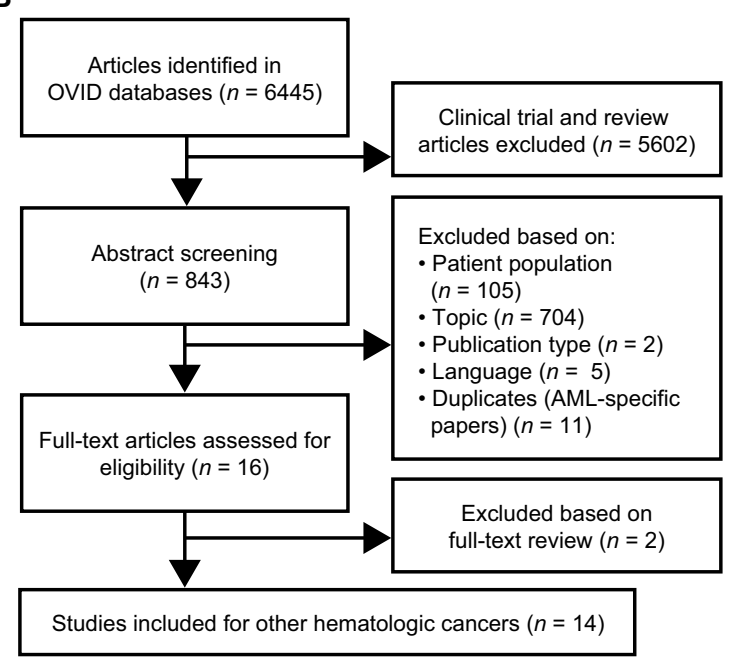

Note: articles could be excluded based on more than one exclusion criteria.

Fig. 1 Literature search results. a Acute myeloid leukemia (AML) and b other hematologic cancers 


\subsection{Patient Experience Following Diagnosis}

Several aspects of the patient experience following diagnosis were raised and discussed (Table 1). A key area of impact on both patients and caregivers was the emotional/ psychological distress of their AML or hematologic cancer diagnosis. In both YouTube videos and literature review articles, patients and caregivers highlighted that they felt uncertain about life expectancy and prognosis, and that death was imminent soon after diagnosis. As a consequence of these uncertainties, there was a strong sense among patients and caregivers that it was not worth planning too far in advance [32-36, 43, 46, 55].

Another common theme was the worries and frustrations experienced by patients following their diagnosis. These ranged from dealing with the side effects of treatment to the impact of their diagnosis on personal relationships. From literature review sources, some patients reported strains on their existing relationships or expressed worries that their diagnosis would prevent the possibility of future relationships [38-40]. In tandem with these concerns was a prevailing need to complete specific tasks before they died, but also a reprioritizing on who or what was important to them, e.g., their career or making amends with family members $[38,41]$.

As expected, the patient experience is complex, particularly in patients with AML. In both data sources, patients with AML described how they experience a lack of energy or willingness to fight their condition, and this was associated with feelings of guilt [37]. However, many patients also detailed their acceptance of their AML diagnosis and how they strive to have a positive disease outlook and retain a sense of normality in their everyday lives. Across patients with hematologic cancer, there was a stronger appreciation for living in the present, and both patients and caregivers expressed gratitude for having remaining time with their loved ones [34, 42-44].

\subsection{Achievement of Life Milestones}

Generally, and from both YouTube videos and literature review sources, patients and caregivers focused on the perceived benefits associated with living longer, and in particular, reaching life milestones (Table 2). Life milestones included reaching the next birthday and a desire to be alive for family occasions, e.g., weddings or births. Younger caregivers found it especially hard to accept the prognosis of AML or other hematologic cancers. They felt there were many important milestones that their spouse or parent would miss out on. Additionally, parents of younger children seemed more anxious about leaving their children, especially those children who had major life events ahead of them [36, $39,40,44,55]$.

As reported in some articles, patients would often set goals to cope with the uncertainty of the disease, and these goals usually revolved around children or grandchildren. Many patients expressed their gratitude at having more time with their family, being able to attend family events, and/or reaching a life milestone $[39,41,42]$.

\subsection{Treatment Decision Making}

Decisions for treatments were often governed by clinical factors (Table 3). In both YouTube videos and the literature review, the patient age, comorbidities, and tolerability issues were all factors reported as having prohibited patients with AML from receiving intensive, potentially life-extending chemotherapy. For example, ineffectiveness of prior
Fig. 2 Staggered approach for searching YouTube. $A M L$ acute myeloid leukemia

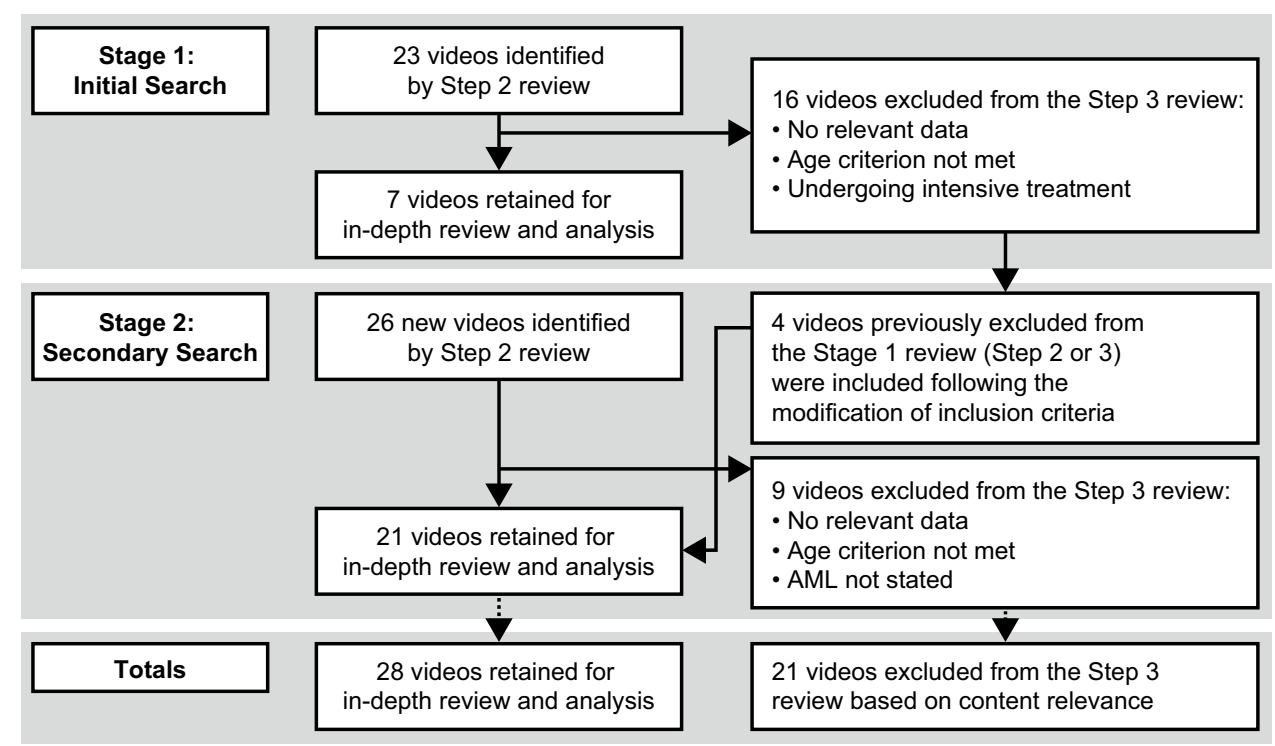




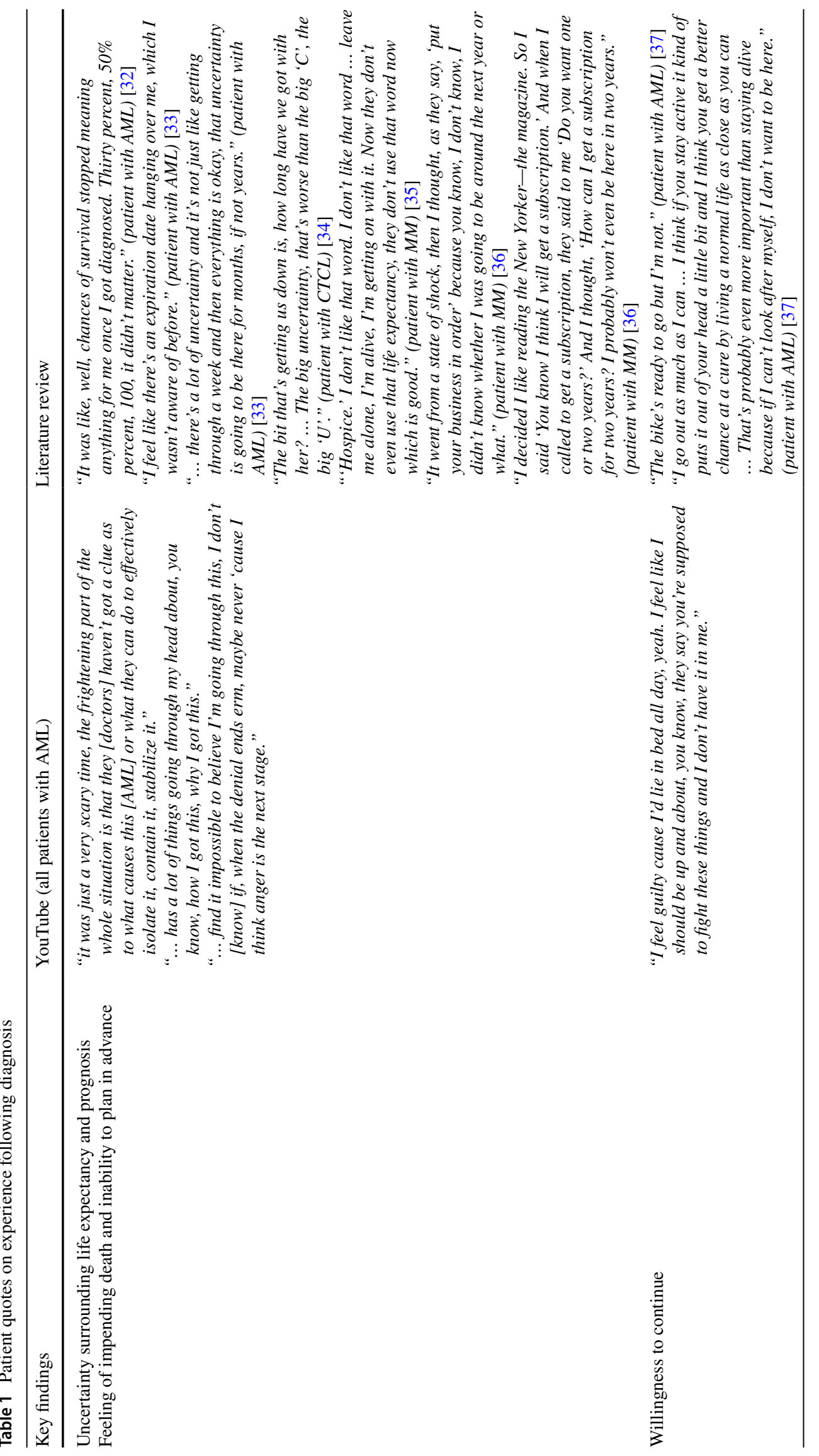




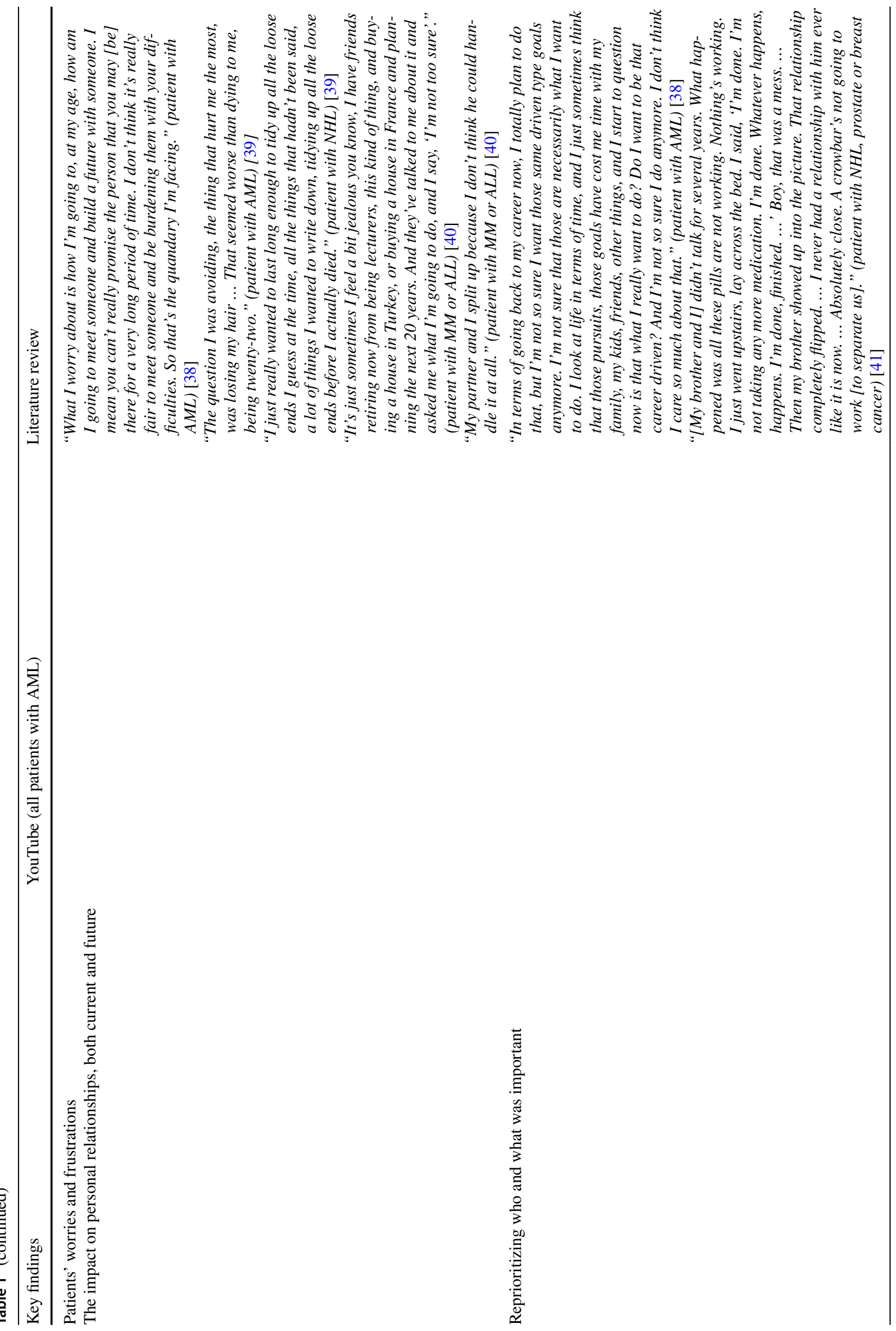




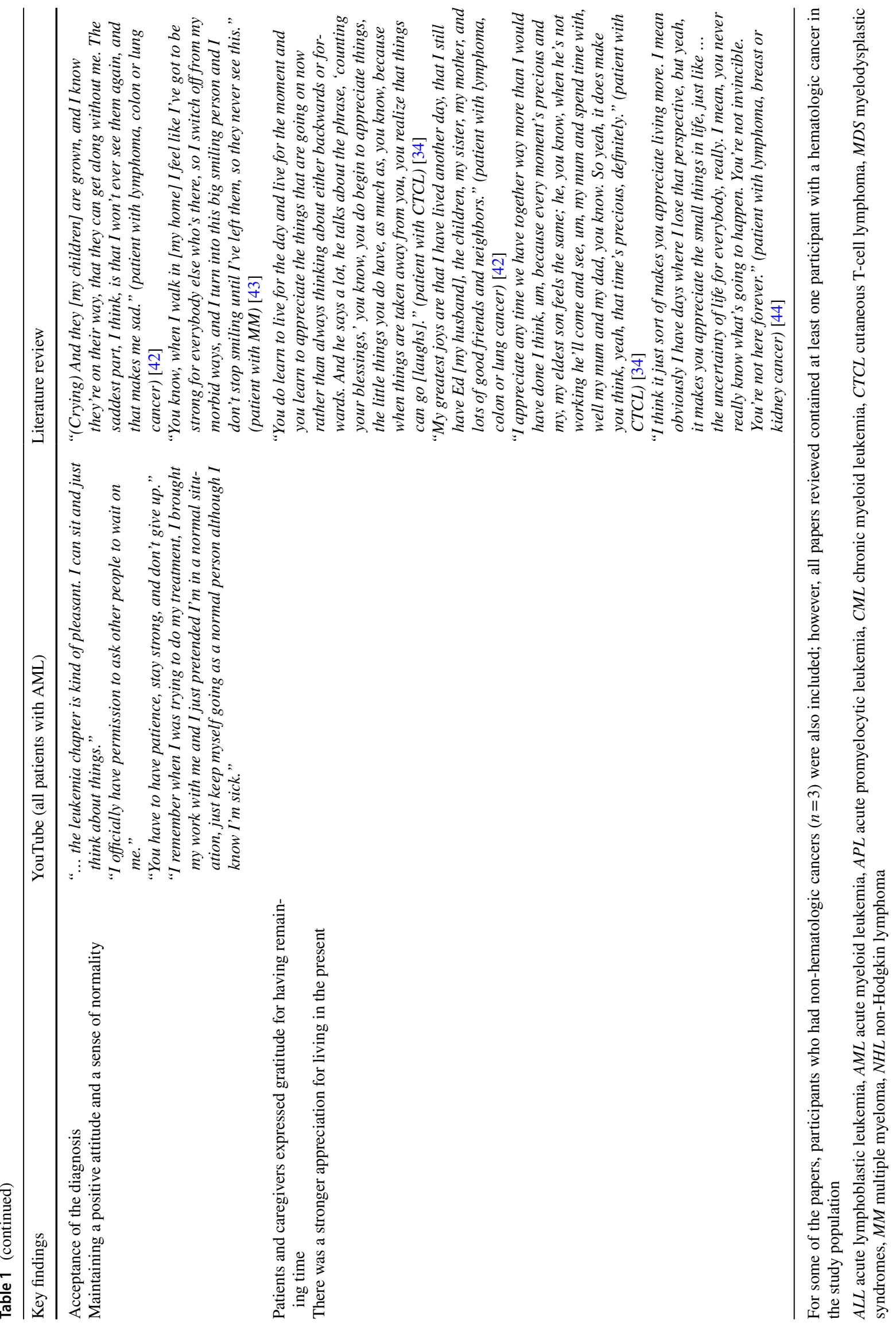


treatments and side effects such as hallucinations and loss of appetite were some of the reasons cited for not receiving intensive chemotherapy.

Patients in both data sources also discussed the lack of a clear defined treatment pathway and felt resentful that there was no 'exact' treatment for AML or hematologic cancer. Additionally, there was a patient-perceived ineffectiveness of chemotherapy, and expectations regarding the success of treatment differed from clinical estimations of survival time. For example, for some patients where prior therapies had failed, they did not want to be too hopeful that a promising treatment would be effective $[33,36,45]$. In parallel, some patients expressed their gratitude to scientific research for prolonging their lives and remained hopeful that there would be new and better treatments in the future for AML or other hematologic cancers.

For patients in both data sources who were eligible for chemotherapy, concern over side effects, tolerability issues, and stress related to the aggressive treatments and procedures were common reasons for their decision not to receive treatment. Another key feature of treatment choice was patients' willingness to undergo or continue treatment; some patients reported they no longer had the energy or fortitude to undergo intensive treatments $[32,47-49,52$, 54]. Whereas, in one article, other patients wanted treatment right up until the very end of life [47]. Among patients with AML, many described feeling trapped between two undesirable treatment choices-intensive chemotherapy vs. palliative or best supportive care; choosing intensive chemotherapy to extend their life was accompanied with the acceptance of potentially distressing side effects associated with this treatment [36, 40, 41, 46-48, 54].

Health-related QoL was an important consideration for patients, in addition to the quantity or length of life [36, 41, 46, 47, 51, 53]. One AML-specific article reported that $97 \%$ of patients agreed with the statement that HRQoL was more important to them than the length of their life, regardless of their choice of therapy [51]. Patients with AML or other hematologic cancers emphasized the importance of maintaining a 'normal life' following their diagnosis and, in particular, the ability to engage in their hobbies and daily activities. However, effective therapies with the potential to extend life were of greater importance to patients/caregivers and for some patients, it outweighed potentially severe side effects [36, 40, 41, 47]. As a result, many patients eligible to receive intensive chemotherapy chose this option over low-intensity treatments.

\subsection{Importance of Treatment Experience}

Patients with AML or other hematologic cancer detailed a number of other aspects of their treatment as being important factors in their HRQoL and daily activities (Table 4).
In both the YouTube videos and literature review sources, patients reported that extended time spent in the hospital and traveling for medical appointments had a detrimental impact on their finances and family life; patients often spent time away from their families and missed out on activities with friends or family. Additionally, patients and caregivers (in the literature review only) felt that they could not make plans because of appointments [32, 34, 36, 37, 40, 43, 48, 50, 52, 53, 56, 57].

Treatment offered at local centers generally helped patients to live a normal family life and minimized the burden to their families associated with extensive and frequent hospital stays. Furthermore, treatment at local centers was beneficial for patients who did not have caregivers or patients with caregivers who were unable to support them through treatment [50]. In some articles, patients expressed concern that most of their remaining time would be spent in a hospital or institution [36, 49]. However, in both data sources, some patients highlighted the value of hospice care in terms of reducing the burden on caregivers. Among patients with AML in the YouTube videos, treatment to counteract side effects was highlighted as a key aspect of managing their condition. Other aspects of treatment commented on by patients with hematologic cancers in the literature review were transfusion dependence and the threat of infections. Both outcomes caused patients to feel trapped either in the hospital or their home [37-39, 46, 47].

\subsection{Intensive Chemotherapy vs. Non-intensive Chemotherapy or Palliative Care}

Some articles and YouTube videos reported findings from an intensive chemotherapy vs. non-intensive treatment perspective [33, 40, 41, 46-48, 51, 54]. One article, focused on treatment decision making in patients with AML who were aged $>60$ years, reported that patients choosing intensive chemotherapy were significantly younger than those who opted for non-intensive treatments (median age: 66 vs. 76 years; $p=0.01$ ) [51]. Furthermore, patients who chose intensive chemotherapy were more likely to report having their treatment decisions influenced by their physician [51]. In the YouTube videos, there were some differences between the intensive chemotherapy patient/caregiver posts and those posted by patients/caregivers of patients receiving non-intensive chemotherapy or palliative care. The foci of posts from the intensive chemotherapy group centered on the positive aspects of living longer; specifically, the return to normal family life, resuming work life, being able to travel, and being able to help other people. In contrast, patients receiving non-intensive chemotherapy/palliative care accepted their prognosis, felt death was inescapable, and treatment was only prolonging the inevitable. 


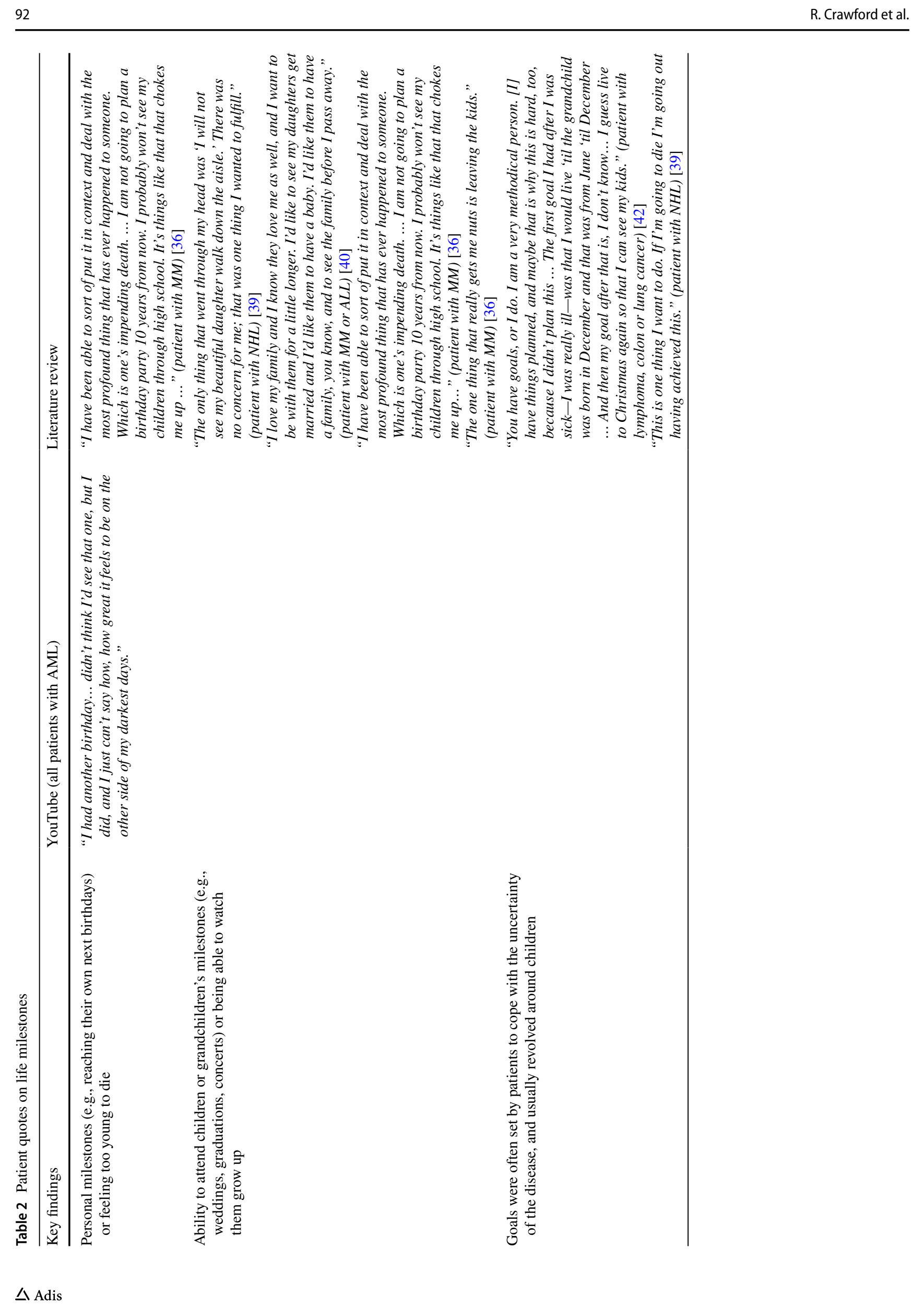




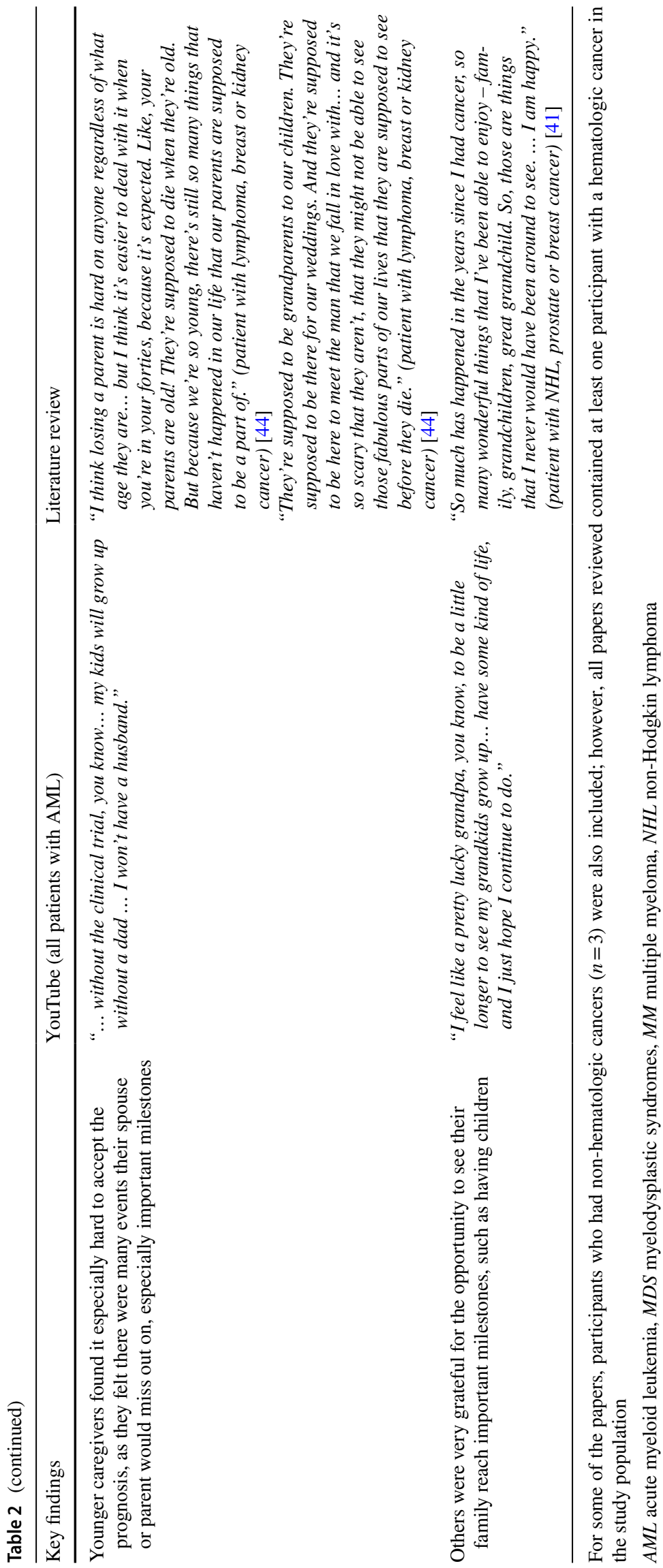




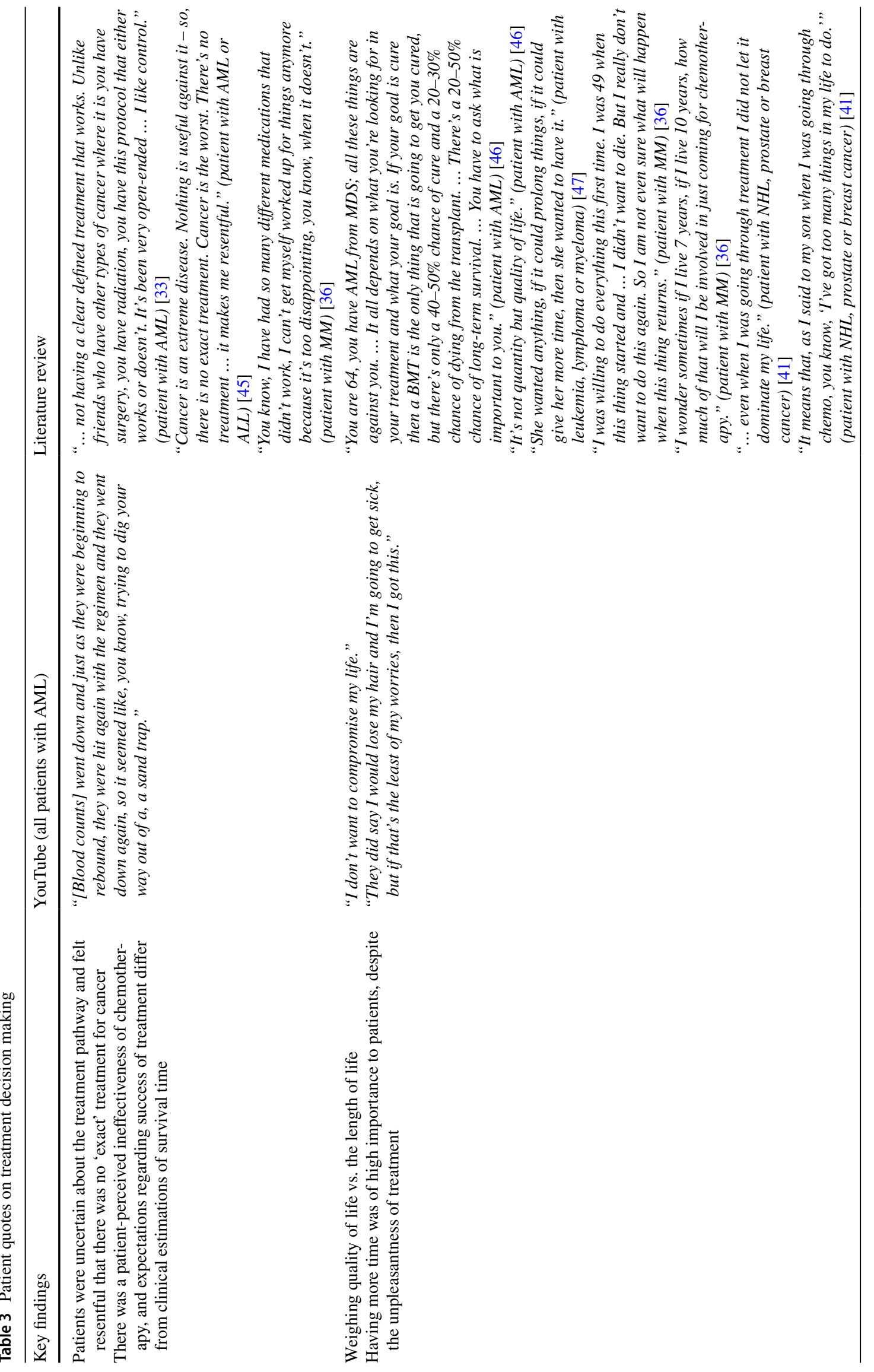




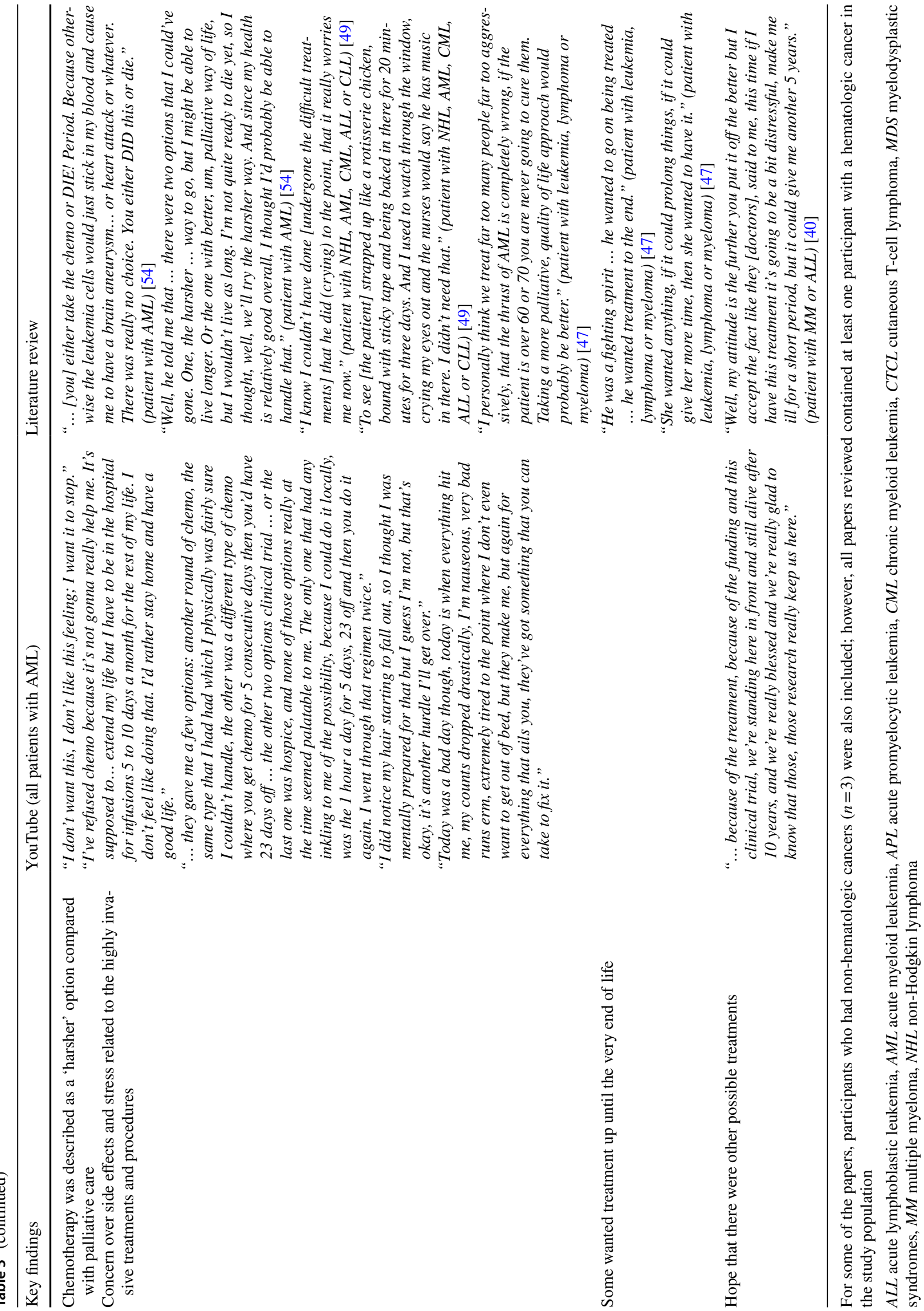




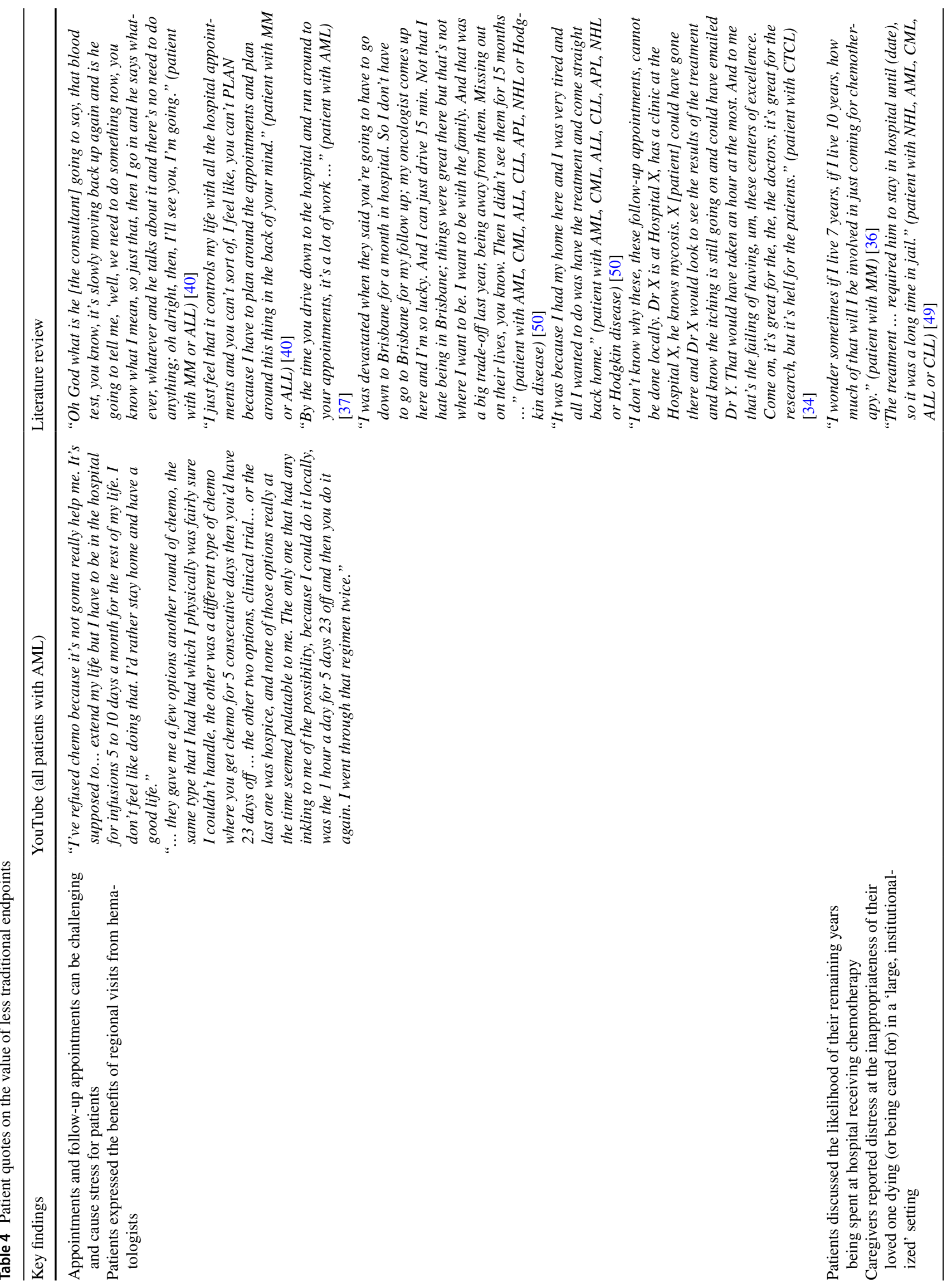




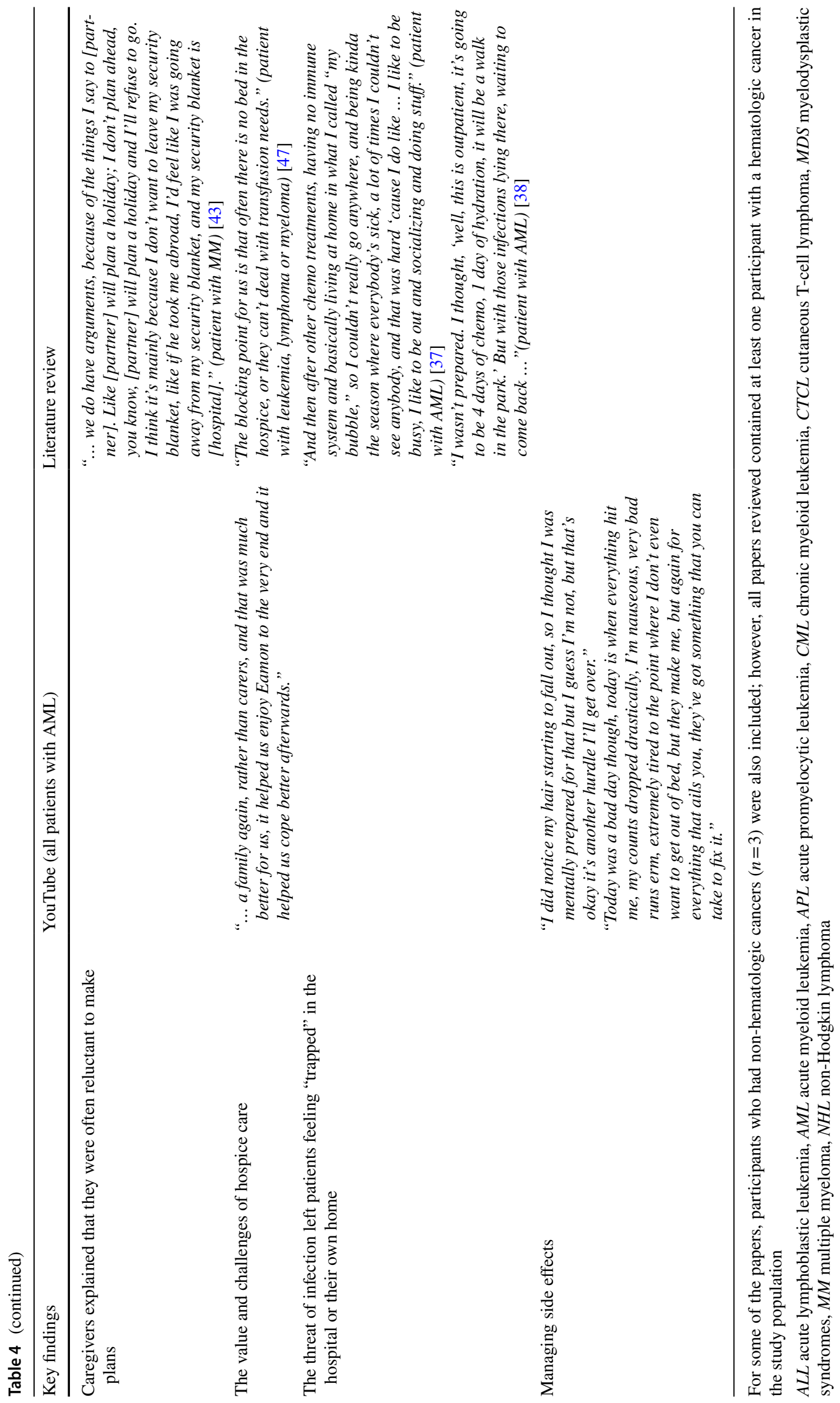




\section{Discussion}

These analyses had originally aimed to focus on patients with AML, but because of the limited availability of published literature within AML, the literature review was expanded to include other hematologic cancers. Both the literature review and YouTube video searches yielded valuable and informative insights on the patient experience and perceptions of hematologic cancers, in particular for AML. There was a large overlap in the key messages and concepts reported by patients using each approach, thus supporting the value of social media searches as a supplement to traditional research methods.

Although patients and caregivers were cognizant of the terminal prognosis of their cancer, most were focused on the possibility of identifying life-extending treatments that would give the patient the opportunity to reach personal milestones and attend family events. Although many patients and caregivers reported a drive to continue living by using any treatments available, other patients were more accepting of their prognosis. The latter group wanted to enjoy their remaining time without the disruption of treatment regimens or the detrimental impact of treatments on HRQoL.

Many patients expressed their worries and frustrations regarding the lack of treatment options and/or a clear treatment pathway, the possibility of side effects, and how their diagnosis and any treatments would affect their relationships, daily lives, and ability to complete certain tasks. The convenience of treatment center locations emerged as a key factor for patients and caregivers-provision of a facility that would allow patients to live a normal life with their family and in close proximity to hospital was considered highly advantageous for patients. Although data were limited, the results highlighted some differences between patients eligible for or receiving intensive chemotherapy vs. those who were not. In general, patients choosing intensive chemotherapy were younger (median age: 66 vs. 76 years; $p=0.01$ ) [51], were more likely to report having had treatment decisions influenced by their physician, and were more focused on the positive aspects of living longer, such as added time with loved ones. Further investigation of the differences in views between patients receiving intensive chemotherapy vs. non-intensive chemotherapy/palliative care will be crucial to understanding treatment decision making from the patient and physician perspective. Because patient choice and treatment objectives are of paramount importance in selecting therapies, establishing patient perspectives on nonintensive chemotherapy/palliative care vs. intensive chemotherapy could improve patient education surrounding treatment options, the treatment pathway, and supportive care.

The patient perspectives gained in the current analyses support the findings from prior studies exploring perspectives and HRQoL among patients with AML, as well as the insights from patient advocacy groups [16-18, 58]. A common theme in the current study was that the emotional/ psychological effect of the diagnosis can have a large impact on the patient, their relationships, and their everyday lives. Other common factors that featured heavily were treatment decision making and the impact of treatments, particularly side effects and the aggressiveness of treatments. In many clinical trials and treatment decisions in clinical practice, there is still a focus on survival as the primary objective of treatment, as opposed to other, less traditional endpoints valued by patients. Treatments that lead to a small increase in overall survival may not be perceived as being efficacious from a clinical, regulatory, or payer perspective; however, prolongation of life, even if only by a few months, is important to many patients. Furthermore, the current analyses showed that patients were willing to accept side effects to potentially prolong life; however, this needed to be considered alongside the patients' desire to retain a normal life and spend time with family/friends, as well as the impact of treatment schedules, the availability of supportive care medication, and the location of treatment centers. Further understanding these insights into patient perspectives and the patient experience could aid discussions between clinicians, patients, and their caregivers regarding treatment decisions, patient management options, and supportive care.

Given the recent US Food and Drug Administration guidelines [22] and the increasing use of social networking websites as a platform to disseminate experience-based information [21], this approach of combining a targeted literature search with a social media review is both novel and timely. The utilization of YouTube for conveying health information and information pertaining to medical treatments has been previously explored in a number of other therapy areas, such as cardiovascular conditions, prostate cancer, and Alzheimer's disease, with varying results [26-31]. However, none of these studies specifically explored whether YouTube searches could be used as a method for collecting patient-centered information to gain insight into the patient disease and treatment experience.

Patient experience data provide an opportunity to explore patients' perspectives on current and potential treatments, with broad clinical, scientific, and regulatory ramifications $[20,22]$. Furthermore, patient-reported information shared on social networking websites, such as YouTube, provides access to unsolicited, publicly available data that can be used to gain insight into the patient experience in the rare and orphan disease setting. In the absence of published literature, these data can inform clinicians, healthcare providers, and payers on the perceptions and needs related to treatments [21]. Gaining patient narratives on the expectation, tolerance, and attitudes towards treatments can enhance clinical management decisions, especially in conditions where there 
is no clear prescribed treatment pathway, e.g., AML and other hematologic cancers. This contextualization has the potential to guide medical product development by highlighting outcomes of importance to patients, leading to improved clinical trial enrollment and retention, and more informed discussions with payers and regulatory agencies [20-22].

In some countries, patient-reported outcomes are becoming increasingly important in reimbursement decision making; however, these can often focus on the proximal signs of disease (e.g., symptoms) and general HRQoL. Therefore, it will be critical to ensure that broader outcomes and patientreported information are incorporated into clinical trials and that more efforts are made to educate clinicians, healthcare providers, and payers on the patient experience and patientreported information, and how they can add value to healthcare and reimbursement decision making [59]. For example, when reviewing clinical data, clinicians, payers and/ or regulatory agencies may not view an increase in overall survival of a few months as being clinically meaningful; however, therapies that could potentially extend life were of high importance to many patients. For patients with terminal prognoses, treatment decisions are based on a careful assessment of potential life expectancy, QoL, and financial burden that is specific to the circumstances of individual patients and their families, e.g., a short-term gain in survival may allow the patient to achieve certain milestones. Therefore, it is important for clinicians, payers, and agencies to be aware that shared decision making and discussions on QoL are highly personal, and these decisions should be less focused on statistical probabilities and more focused on patient preferences and values [60]. As decision makers, regulatory and reimbursement agencies increasingly rely on multi-criteria decision analysis frameworks, the ability to gather and compile meaningful patient-centric input is poised to become instrumental to achieve holistic healthcare decisions that are both scientifically robust and societally acceptable [61].

The combination of a targeted literature review with YouTube data is a novel approach to obtaining patient perspectives in hematological cancers, and in particular, AML; however, there are a few limitations to these analyses. Of the articles reviewed in the targeted literature search, a limited number focused on older adults with AML, a particular population of interest. As such, the search had to be widened to all patients with AML; however, this approach helped to provide a wider context and understanding of the condition. Additionally, the articles reviewed were published from 1986 to 2018 and therefore some of the reviewed articles may not reflect current opinion on treatment options for patients with AML. The treatment landscape for AML has changed significantly in recent years and continues to evolve (with the approval of five new drugs in 2017-2018); therefore, it is unlikely these treatments were captured in the reviewed articles and YouTube videos. This highlights the evidence gap and unmet need for this type of research to reflect the current management of patients with AML, and illustrates that patient perspectives on these newly approved therapies should be a target for future studies. Three of the articles were conference abstracts and not full journal articles, thus limiting the level of information to be extracted.

Furthermore, there are some limitations to using social media: data are unsolicited and not generated to answer specific research questions. However, this could also be perceived as a strength in that they highlight the most important aspects of the patient experience and are free from research bias. The content is also not regulated or peer reviewed, and users can upload any content they choose, i.e., relevant content is often embedded within irrelevant narratives. In addition, YouTube does not allow for the implementation of a sophisticated or comprehensive search strategy, thus the researcher is limited to searches using simple search terms or simple Boolean operators that may or may not identify all relevant content. There are also limitations in terms of sampling, particularly self-selection bias and reliance on patient self-identification and diagnosis, which may not be verifiable. Patient-reported age was not always available, thus the use of a researcher-determined lower age boundary (i.e., 60 years) could be open to error. However, steps were taken to minimize this researcher bias as far as possible by using two independent researchers to determine a lower age boundary and a third researcher to resolve any disagreement. The quantity of data may be limited following review and may only be from a select group of individuals; however, this is a consequence of the unsolicited and spontaneous nature of the data. Additionally, content may be removed from YouTube because of copyright issues, upon the creator's request or by caregivers when the patient has died. Furthermore, different social media platforms (e.g., Facebook, Instagram) are used by different demographic groups, and there may be a potential bias toward younger patient populations who are more engaged with social media platforms.

Although there are limitations associated with the use of social media, it still offers valuable data sources that can provide snapshots into patient priorities and longitudinal data on the patient-lived experience that may not be captured by traditional methods, e.g., interviews and surveys. Furthermore, YouTube has several advantages as a source of data: data are easily accessible, patient-reporting burden is negligible, there is a lack of interviewer bias, and there are minimal recall issues. Additionally, social media is an existing source of data generated independently by individual users; it does not have the resource burdens typically associated with more traditional data collection methods (e.g., face-to-face interviews). This is particularly salient for research concerning patients with rare diseases who may be difficult to access and potentially geographically dispersed. 
Accessing the patient/caregiver-lived experience of rare diseases via social media can generate initial information to justify the costs of qualitative research, or can provide a valuable adjunct to qualitative research where sample sizes for hard-to-reach populations are likely to be small.

\section{Conclusions}

In summary, these analyses support the value of YouTube video searches as a supplement to a targeted literature review. Both analyses provided complementary and valuable information on the patient experience and perceptions of hematologic cancers, in particular for AML. Some treatments may not be considered efficacious from a clinical, regulatory, or payer perspective, as they only confer small increases in overall survival, but they may be clinically meaningful to patients because they allow the patient to have additional time with their loved ones and to achieve life milestones. The patient experience is complex and multifactorial, thus patient management and treatment decisions in clinical practice need to reflect the expectations, goals, and preferences for a given individual. This is particularly true in rare disease/orphan indications and conditions with no clear prescribed treatment pathway, where patient-reported information can contextualize joint treatment decisions made by patients and their treating physicians, enhance clinical management/guidelines and payer decision making, and inform medical product development, including providing input into clinical trial design and interpretation.

Acknowledgements The authors thank Megan Latham for her assistance with the targeted literature review. Medical writing support was provided by Anne Marie McGonigal, PhD, of Engage Scientific Solutions, and funded by Pfizer Inc.

Author Contributions All authors were involved in the trial conception/ design, or the acquisition, analysis, or interpretation of data. All authors contributed to the drafting of the manuscript and approved the final version. All authors contributed equally to the creation of this manuscript.

\section{Compliance with Ethical Standards}

Funding These analyses were sponsored by Pfizer Inc.

Conflict of interest Rebecca Crawford, Rebecca Conroy, and Lynda Doward are employees of RTI Health Solutions, which received funding from Pfizer Inc. for these analyses. Timothy Bell, Verna Welch, and Francois Peloquin are employees of Pfizer Inc. Chloe Johnson, Kate Sully, and Adam Gater are employees of Adelphi Values, which received funding from Pfizer Inc. for these analyses.

Ethics approval and consent to participate Prior to data collection, an institutional review board committee reviewed the YouTube study and determined that the social media review did not constitute research with human subjects. Ethics approval was deemed unnecessary as the study data were located within the public domain. Ethics approval was not required for the literature review study.

Data availability The datasets generated from YouTube and targeted literature review resources and analyzed for the current study are available from the corresponding author on reasonable request.

Open Access This article is distributed under the terms of the Creative Commons Attribution-NonCommercial 4.0 International License (http://creativecommons.org/licenses/by-nc/4.0/), which permits any noncommercial use, distribution, and reproduction in any medium, provided you give appropriate credit to the original author(s) and the source, provide a link to the Creative Commons license, and indicate if changes were made.

\section{References}

1. De Kouchkovsky I, Abdul-Hay M. Acute myeloid leukemia: a comprehensive review and 2016 update. Blood Cancer J. 2016;6:e441.

2. Finn L, Dalovisio A, Foran J. Older patients with acute myeloid leukemia: treatment challenges and future directions. Ochsner J. 2017;17:398-404.

3. Bell JA, Galaznik A, Huelin R, Stokes M, Guo Y, Fram RJ, et al. Effectiveness and safety of therapeutic regimens for elderly patients with acute myeloid leukemia: a systematic literature review. Clin Lymphoma Myeloma Leuk. 2018;18:e303-14.

4. Burnett A, Wetzler M, Löwenberg B. Therapeutic advances in acute myeloid leukemia. J Clin Oncol. 2011;29:487-94.

5. Bower H, Andersson TM, Björkholm M, Dickman PW, Lambert PC, Derolf AR. Continued improvement in survival of acute myeloid leukemia patients: an application of the loss in expectation of life. Blood Cancer J. 2016;6:e390.

6. Bhayat F, Das-Gupta E, Smith C, McKeever T, Hubbard R. The incidence of and mortality from leukaemias in the UK: a general population-based study. BMC Cancer. 2009;9:252.

7. Juliusson G, Antunovic P, Derolf A, Lehmann S, Mollgard L, Stockelberg D, et al. Age and acute myeloid leukemia: real world data on decision to treat and outcomes from the Swedish Acute Leukemia Registry. Blood. 2009;113:4179-87.

8. Maynadié M, Girodon F, Manivet-Janoray I, Mounier M, Mugneret F, Bailly F, et al. Twenty-five years of epidemiological recording on myeloid malignancies: data from the specialized registry of hematologic malignancies of Cote d'Or (Burgundy, France). Haematologica. 2011;96:55-61.

9. Matsunaga T, Yamashita K, Kubuki Y, Toyama T, Imataki O, Maeda K, et al. Acute myeloid leukemia in clinical practice: a retrospective population-based cohort study in Miyazaki Prefecture, Japan. Int J Hematol. 2012;96:342-9.

10. Shah BK, Ghimire KB. Improved survival among older acute myeloid leukemia patients: a population-based study. Acta Oncol. 2014;53:935-8.

11. Osca-Gelis G, Puig-Vives M, Saez M, Gallardo D, Lloveras $\mathrm{N}$, Guardia $\mathrm{R}$, et al. Is survival in myeloid malignancies really improving? A retrospective 15-year population-based study. Leuk Lymphoma. 2015;56:896-902.

12. Thein MS, Ershler WB, Jemal A, Yates JW, Baer MR. Outcome of older patients with acute myeloid leukemia: an analysis of SEER data over 3 decades. Cancer. 2013;119:2720-7.

13. Oran B, Weisdorf DJ. Survival for older patients with acute myeloid leukemia: a population-based study. Haematologica. 2012;97:1916-24. 
14. Yogaparan T, Panju A, Minden M, Brandwein J, Mohamedali $\mathrm{HZ}$, Alibhai SM. Information needs of adult patients 50 or older with newly diagnosed acute myeloid leukemia. Leuk Res. 2009;33:1288-90.

15. Alibhai SM, Breunis H, Timilshina N, Brignardello-Petersen R, Tomlinson G, Mohamedali H, et al. Quality of life and physical function in adults treated with intensive chemotherapy for acute myeloid leukemia improve over time independent of age. J Geriatr Oncol. 2015;6:262-71.

16. Korol EE, Wang S, Johnston K, Ravandi-Kashani F, Levis M, van Nooten F. Health-related quality of life of patients with acute myeloid leukemia: a systematic literature review. Oncol Ther. 2017;5:1-16.

17. Bryant AL, Deal AM, Battaglini CL, Phillips B, Pergolotti M, Coffman E, et al. The effects of exercise on patient-reported outcomes and performance-based physical function in adults with acute leukemia undergoing induction therapy: Exercise and Quality of Life in Acute Leukemia (EQUAL). Integr Cancer Ther. 2018;17:263-70.

18. van Nooten F, Korol EE, Levis M, Ravandi-Kashani F, Wang S, Johnston K. Health-related quality of life of patients with acute myeloid leukemia: a systematic literature review. Value Health. 2016;19:A160.

19. Forsythe A, Kwon CS, Bell T, Smith TA, Arondekar B. Healthrelated quality of life in acute myeloid leukemia patients not eligible for intensive chemotherapy: results of a systematic literature review. Clinicoecon Outcomes Res. 2019;11:87-98.

20. Forsythe L, Heckert A, Margolis MK, Schrandt S, Frank L. Methods and impact of engagement in research, from theory to practice and back again: early findings from the Patient-Centered Outcomes Research Institute. Qual Life Res. 2018;27:17-31.

21. Baldwin M, Spong A, Doward L, Gnanasakthy A. Patient-reported outcomes, patient-reported information: from randomized controlled trials to the social web and beyond. Patient. 2011;4:11-7.

22. US Food and Drug Administration. Patient-focused drug development: collecting comprehensive and representative input. Guidance for industry, Food and Drug Administration staff, and other stakeholders. Draft guidance. https://www.fda.gov/downloads/ Drugs/GuidanceComplianceRegulatoryInformation/Guidances/ UCM610442.pdf. Accessed 20 Aug 2019.

23. McDonald L, Malcolm B, Ramagopalan S, Syrad H. Real-world data and the patient perspective: the PROmise of social media? BMC Med. 2019;17:11

24. Rothman M, Gnanaskathy A, Wicks P, Papadopoulos EJ. Can we use social media to support content validity of patient-reported outcome instruments in medical product development? Value Health. 2015;18:1-4.

25. McCarrier KP, Bull S, Fleming S, Simacek K, Wicks P, Cella $D$, et al. Concept elicitation within patient-powered research networks: a feasibility study in chronic lymphocytic leukemia. Value Health. 2016;19:42-52.

26. Chen HM, Hu ZK, Zheng XL, Yuan ZS, Xu ZB, Yuan LQ, et al. Effectiveness of YouTube as a source of medical information on heart transplantation. Interact J Med Res. 2013;2:e28.

27. Kwok TM, Singla AA, Phang K, Lau AY. YouTube as a source of patient information for varicose vein treatment options. J Vasc Surg Venous Lymphat Disord. 2017;5:238-43.

28. Pant S, Deshmukh A, Murugiah K, Kumar G, Sachdeva R, Mehta JL. Assessing the credibility of the "YouTube approach" to health information on acute myocardial infarction. Clin Cardiol. 2012;35:281-5.

29. Steinberg PL, Wason S, Stern JM, Deters L, Kowal B, Seigne J. YouTube as source of prostate cancer information. Urology. 2010;75:619-22.
30. Tang W, Olscamp K, Choi SK, Friedman DB. Alzheimer's disease in social media: content analysis of YouTube videos. Interact J Med Res. 2017;6:e19.

31. Lee JL, Frey M, Frey P, Hollin IL, Wu AW. Seeing is engaging: vlogs as a tool for patient engagement. Patient. 2017;10:267-70.

32. Bridges JF, Oakes AH, Reinhart CA, Voyard E, O'Donoghue B. Developing and piloting an instrument to prioritize the worries of patients with acute myeloid leukemia. Patient Prefer Adherence. 2018;12:647-55.

33. Boucher NA, Johnson KS, LeBlanc TW. Acute leukemia patients' needs: qualitative findings and opportunities for early palliative care. J Pain Symptom Manag. 2018;55:433-9.

34. Selman LE, Beynon T, Radcliffe E, Whittaker S, Orlowska D, Child F, et al. 'We're all carrying a burden that we're not sharing': a qualitative study of the impact of cutaneous T-cell lymphoma on the family. Br J Dermatol. 2015;172:1581-92.

35. McGrath P. End-of-life care in hematology: update from Australia. J Soc Work End Life Palliat Care. 2013;9:96-110.

36. Dahan JF, Auerbach CF. A qualitative study of the trauma and posttraumatic growth of multiple myeloma patients treated with peripheral blood stem cell transplant. Palliat Support Care. 2006;4:365-87.

37. Deckert AL, Gheihman G, Nissim R, Chung C, Schimmer AD, Zimmermann $\mathrm{C}$, et al. The importance of meaningful activity in people living with acute myeloid leukemia. Leuk Res. 2018;67:86-91.

38. Nissim R, Rodin G, Schimmer A, Minden M, Rydall A, Yuen D, et al. Finding new bearings: a qualitative study on the transition from inpatient to ambulatory care of patients with acute myeloid leukemia. Support Care Cancer. 2014;22:2435-43.

39. Xuereb MC, Dunlop R. The experience of leukaemia and bone marrow transplant: searching for meaning and agency. Psychooncology. 2003;12:397-409.

40. Maher K, de Vries K. An exploration of the lived experiences of individuals with relapsed multiple myeloma. Eur J Cancer Care (Engl). 2011;20:267-75.

41. Hannum SM, Clegg Smith K, Coa K, Klassen AC. Identity reconstruction among older cancer survivors: age and meaning in the context of a life-altering illness. J Psychosoc Oncol. 2016;34:477-92.

42. Mackey KM, Sparling JW. Experiences of older women with cancer receiving hospice care: significance for physical therapy. Phys Ther. 2000;80:459-68.

43. Molassiotis A, Wilson B, Blair S, Howe T, Cavet J. Living with multiple myeloma: experiences of patients and their informal caregivers. Support Care Cancer. 2011;19:101-11.

44. Puterman J, Cadell S. Timing is everything: the experience of parental cancer for young adult daughters: a pilot study. J Psychosoc Oncol. 2008;26:103-21.

45. Farsi $Z$. The meaning of disease and spiritual responses to stressors in adults with acute leukemia undergoing hematopoietic stem cell transplantation. J Nurs Res. 2015;23:290-7.

46. Crighton MH, Lingler JH, Happ MB. Fit and misfit. Res Gerontol Nurs. 2011;4:27-35.

47. McCaughan D, Roman E, Smith AG, Garry A, Johnson M, Patmore R, et al. Determinants of hospital death in haematological cancers: findings from a qualitative study. BMJ Support Palliat Care. 2018;8:78-86.

48. LeBlanc TW, Fish LJ, Bloom CT, El-Jawahri A, Davis DM, Locke $\mathrm{SC}$, et al. Patient experiences of acute myeloid leukemia (AML): a qualitative study about diagnosis, illness understanding, and treatment decision-making. Blood. 2015;126:2119.

49. McGrath P. Dying in the curative system: the haematology/oncology dilemma. Part 1. Aust J Holist Nurs. 2001;8:22-30. 
50. McGrath P. Overcoming the distance barrier in relation to treatment for haematology patients: Queensland findings. Aust Health Rev. 2015;39:344-50.

51. Sekeres MA, Stone RM, Zahrieh D, Neuberg D, Morrison V, De Angelo DJ, et al. Decision-making and quality of life in older adults with acute myeloid leukemia or advanced myelodysplastic syndrome. Leukemia. 2004;18:809-16.

52. Seo J, Smith BD, Estey E, Voyard E, O’Donoghue B, Bridges JFP. Developing an instrument to assess patient preferences for benefits and risks of treating acute myeloid leukemia to promote patientfocused drug development. Curr Med Res Opin. 2018;34:2031-9.

53. Buckley SA, Jimenez-Sahagun D, Othus M, Walter RB, Lee SJ. Quality of life from the perspective of the patient with acute myeloid leukemia. Cancer. 2018;124:145-52.

54. LeBlanc TW, Fish LJ, Bloom CT, El-Jawahri A, Davis DM, Locke $\mathrm{SC}$, et al. Patient experiences of acute myeloid leukemia: a qualitative study about diagnosis, illness understanding, and treatment decision-making. Psychooncology. 2017;26:2063-8.

55. Bevans M, Sternberg EM. Caregiving burden, stress, and health effects among family caregivers of adult cancer patients. JAMA. 2012;307:398-403.

56. Orlowska D, Selman LE, Beynon T, Radcliffe E, Whittaker S, Child F, Harding R. 'It's a traumatic illness, traumatic to witness': a qualitative study of the experiences of bereaved family caregivers of patients with cutaneous T-cell lymphoma. Br J Dermatol. 2018;179:882-8.

57. Cran F, Poynton C, Harness M. Quality of life in patients over 60 with acute myeloid leukaemia on palliative care pathways. In: 49th Annual scientific meeting of the British Society for Haematology; Brighton (UK). Br J Haematol. 2009;145(Suppl. S1): abstract no. 100 .

58. Canadian Agency for Drugs and Technologies in Health. panCanadian Oncology Drug Review: final clinical guidance report. https://www.cadth.ca/sites/default/files/pcodr/pcodr_midostauri n_rydapt_all_fn_cgr.pdf. Accessed 20 Aug 2019.

59. Brogan AP, DeMuro C, Barrett AM, D’Alessio D, Bal V, Hogue SL. Payer perspectives on patient-reported outcomes in health care decision making: oncology examples. J Manag Care Spec Pharm. 2017;23:125-34.

60. Yu PP. Challenges in measuring cost and value in oncology: making it personal. Value Health. 2016;19:520-4.

61. EVIDEM. Evidence and values: impact of decision making. https ://www.evidem.org/wp/wp-content/uploads/2017/09/EVIDE M-10th-Edition-Concept-and-definitions.pdf. Accessed 20 Aug 2019.

\section{Affiliations}

\section{Rebecca Crawford ${ }^{1} \cdot$ Kate Sully $^{2} \cdot$ Rebecca Conroy $^{1} \cdot$ Chloe Johnson $^{2} \cdot$ Lynda Doward $^{1} \cdot$ Timothy Bell $^{3} \cdot$ Verna Welch $^{3}$. Francois Peloquin ${ }^{4} \cdot$ Adam Gater $^{2}$}

\author{
Rebecca Crawford \\ rcrawford@rti.org \\ Kate Sully \\ kate.sully@adelphivalues.com \\ Rebecca Conroy \\ rconroy@rti.org \\ Chloe Johnson \\ chloe.johnson@adelphivalues.com \\ Lynda Doward \\ ldoward@rti.org \\ Verna Welch \\ Verna.Welch@pfizer.com
}

Francois Peloquin

Francois.Peloquin@pfizer.com

Adam Gater

adam.gater@adelphivalues.com

1 RTI Health Solutions, Didsbury, Manchester, UK

2 Adelphi Values Ltd, Macclesfield, Cheshire, UK

3 Pfizer Inc, New York, NY, USA

4 Pfizer Canada Inc, Kirkland, QC, Canada 